\title{
Synthesis and Properties of Gelling Fluoroalkylated End-Capped Oligomers Containing Hydroxy Segments
}

\author{
Hideo Sawada, ${ }^{* * *, \dagger}$ Toshihiro Tanimura, ${ }^{*}$ Shinsuke Katayama, ${ }^{* *}$ Tokuzo Kawase, ${ }^{* * *}$ \\ Toshio Tomita, ${ }^{* * * *}$ and Masanori BaBA $* * * * *$ \\ * Department of Chemistry, Nara National College of Technology, \\ Yamatokoriyama, Nara 639-1080, Japan \\ ** Department of Chemistry, Faculty of Advanced Engineering, Nara National \\ College of Technology, Yamatokoriyama, Nara 639-1080, Japan \\ *** Faculty of Human Life Science, Osaka City University, \\ Sumiyoshi, Osaka 558-8585, Japan \\ **** Faculty of Agriculture, Tohoku University, Tsutsumidori-Amamiya \\ Aoba-ku, Sendai 981-8555, Japan \\ *****Division of Human Retroviruses, Center for Chronic Viral Diseases, \\ Faculty of Medicine, Kagoshima University, Sakuragaoka, \\ Kagoshima 890-8520, Japan
}

(Received January 14, 1998)

\begin{abstract}
Reactions of fluoroalkanoyl peroxides with 3-methacryloxy-2-hydroxypropyltrimethylammonium chloride (MHPTA) or 2-hydroxy-2-[1-oxoprop-2-enyl)amino]acetic acid (HOPPA) were found to give new fluoroalkylated end-capped oligomers containing 2-hydroxypropyltrimethylammonium or carboxy-hydroxymethylamido segments, respectively, under very mild conditions. These fluoroalkylated end-capped oligomers form a highly viscoelastic fluid ("gel-like") or cause gelation either in water or in polar organic solvents such as $\mathrm{MeOH}, \mathrm{EtOH}, N, N$-dimethylformamide (DMF), and dimethyl sulfoxide (DMSO), whose behavior is governed by the synergistical interactions of strong aggregations of fluoroalkyl segments within oligomers and intermolecular hydrogen bonding between hydroxy or carboxy segments under the non-crosslinked conditions. The fluorinated MHPTA oligomer possessing "gel-like" characteristic was able to reduce the surface tension of water effectively to around $15 \mathrm{~m} \mathrm{~m}^{-1}$ with a clear break point, resembling critical micell concentration (CMC). These fluoroalkylated MHPTA oligomer hydrogels had strong metal ion binding or releasing power, and exhibited antibacterial activity to some extent against Staphylococcus aureus. They should thus have high potential for new fluorinated functional materials owing to possessing not only common fluorinated properties such as surface activity and biological activity, but also gelling ability by the aggregation of fluoroalkyl segments.

KEY WORDS Gelation / Oligomers / Aggregation / Fluoroalkylated End-Groups / Hydroxy Groups / Carboxy Groups / Surface Tension / Metal Ion Binding / Antibacterial Activity /
\end{abstract}

Fluorinated polymers are a very interesting and useful class of materials due to their unique balance of properties such as low surface free energy, low coefficient of friction, and solvent and chemical resistance. ${ }^{1}$ However, these fluorinated compounds in general exhibit extremely low solubility in organic solvents. Therefore, it has been deeply desirable to explore highly soluble fluorinated macromolecules possessing excellent properties imparted by fluorine that set them apart from the usual fluorinated polymers. From such points of view, we found that the partially fluorinated polymeric compounds, especially fluoroalkylated end-capped macromolecules with carbon-carbon bond formation, prepared by using fluoroalkanoyl peroxides as key intermediates, exhibit a high solubility in various solvents and an excellent surface active property imparted by fluorine and biological activities although these polymers have only fluoroalkylated end-capped materials. ${ }^{2}$ Partially fluoroalkylated polymers were prepared by a variety of anionic polymerizations $^{3-6}$ and a fluoroalkylated end-capped moiety was introduced through the ester bond ${ }^{7-9}$ to perfluoroalkyl-terminated polymers, and the interesting properties have been reported. In a series of such fluoroalkylated end-capped macromolecules, we discovered that fluoroalkylated end-capped oligomers con-

\footnotetext{
+ To whom all correspondence should be addressed.
}

taining betaine segments cause a gelation derived from the synergistical interaction of the aggregations of fluoroalkyl segments and ionic interaction between the betaine segments. ${ }^{10}$ Furthermore, in the fluoroalkylated end-capped oligomers containing triol segments, we have found that the hydrogen bonding interaction between triol segments can be participated in the gelator which is constructed by the fluoroalkyl units. ${ }^{11}$ Previously, Tweig et al. reported similar gel formation of lowmolecular semifluorinated alkanes such as $\mathrm{F}\left(\mathrm{CF}_{2}\right)_{10^{-}}$ $\left(\mathrm{CH}_{2}\right)_{12} \mathrm{H}$ in hydrocarbon solvents $\left[\mathrm{H}\left(\mathrm{CH}_{2}\right)_{p} \mathrm{H} ; p=8\right.$, $10,12,14] .{ }^{12}$ However, studies on such interesting gelations of polymeric organofluorine compounds have been hitherto very limited except for our recent reports. ${ }^{10,11}$ Therefore, it is very interesting to explore partially fluorinated polymeric compounds which cause gelation through the aggregation of fluoroalkyl segments.

In a preliminary account, we reported that fluoroalkylated end-capped oligomers containing 2-hydroxypropyltrimethylammonium or carboxy(hydroxy)methylamido segments cause gelations in aqueous and organic media under the non-crosslinked conditions as well as the corresponding oligomers containing betaine or triol segments. ${ }^{13}$ These gelling fluoroalkylated oligomers had a high metal ion binding or releasing power and antibacterial activity. This paper describes the synthesis and properties of fluoroalkylated end-capped oligomers 
containing 2-hydroxypropyltrimethylammonium or carboxy(hydroxy)methylamido segments.

\section{EXPERIMENTAL}

NMR spectra were measured using a Varian Unityplus $500(500 \mathrm{MHz})$ spectrometer, while IR spectra were recorded on a HORIBA FT-300 FT-IR spectrophotometer. Absorption spectra were recorded on a Shimadzu UV-240 spectrophotometer. Solution viscosity was measured by using a falling-sphere Haake Viscometer D1G.

\section{Materials}

A series of fluoroalkanoyl peroxides $\left[\left(\mathrm{R}_{\mathrm{F}} \mathrm{COO}\right)_{2}\right]$ was prepared by reactions of the corresponding acyl halides and hydrogen peroxide in the presence of aqueous sodium hydroxide according to our previously reported method. ${ }^{14}$ 3-Methacryloxy-2-hydroxypropyltrimethylammonium chloride (MHPTA) was supplied by NOF Corporation. 2-Hydroxy-2-[1-oxoprop-2-enyl)amino]acetic acid (HOPPA) was purchased from Acros Organics Inc. Chromium(III) nitrate and cobalt(II) chloride were purchased from Wako Chemicals.

\section{General Procedure for the Synthesis of Fluoroalkylated End-Capped Oligomers}

Perfluorobutyryl peroxide $(4 \mathrm{mmol})$ in $1: 1$ mixed solvents (AK-225) of 1,1-dichloro-2,2,3,3,3-pentafluoropropane and 1,3-dichloro-1,2,2,3,3-pentafluoropropane $(65 \mathrm{~g})$ was added to an aqueous solution $(50 \%, \mathrm{w} / \mathrm{w})$ of MHPTA (42 mmol). The heterogeneous solution was stirred vigorously at $45^{\circ} \mathrm{C}$ for $5 \mathrm{~h}$ under nitrogen. After evaporating the solvent, the crude product was reprecipitated from methanol-tetrahydrofuran system to give bis(perfluoropropylated) 3-methacryloxy-2-hydroxypropyltrimethylammonium chloride oligomers (Run 2 in Table I; $6.48 \mathrm{~g})$. This oligomer showed the following spectral data: IR $\left(\mathrm{cm}^{-1}\right) 3440(\mathrm{OH}), 1716(\mathrm{C}=\mathrm{O}), 1319$ $\left(\mathrm{CF}_{3}\right), 1245\left(\mathrm{CF}_{2}\right) ;{ }^{1} \mathrm{H}$ NMR $\left(\mathrm{D}_{2} \mathrm{O}\right) \delta 0.70-1.10\left(\mathrm{CH}_{3}\right)$, $1.73-2.00\left(\mathrm{CH}_{2}\right), 3.07-3.22\left(\mathrm{CH}_{3}\right), 3.35-3.50\left(\mathrm{CH}_{2}\right)$, $3.80-4.10\left(\mathrm{CH}_{2}\right), 4.40-4.55(\mathrm{CH}) ;{ }^{19} \mathrm{~F} \mathrm{NMR}\left(\mathrm{D}_{2} \mathrm{O}\right.$, ext. $\left.\mathrm{CF}_{3} \mathrm{CO}_{2} \mathrm{H}\right) \delta-5.57(6 \mathrm{~F}),-43.04(4 \mathrm{~F}),-52.80$ (4F).

The other products obtained exhibited the following spectral characteristics:

$\mathrm{R}_{\mathrm{F}}-(\mathrm{MHPTA})_{n}-\mathrm{R}_{\mathrm{F}}\left[\mathrm{R}_{\mathrm{F}}=\mathrm{C}_{3} \mathrm{~F}_{7}\right.$; Run 1 in Table I]; IR $\left(\mathrm{cm}^{-1}\right) 3448(\mathrm{OH}), 1716(\mathrm{C}=\mathrm{O}), 1319\left(\mathrm{CF}_{3}\right), 1245\left(\mathrm{CF}_{2}\right)$; ${ }^{1} \mathrm{H}$ NMR $\left(\mathrm{D}_{2} \mathrm{O}\right) \delta 0.70-1.10\left(\mathrm{CH}_{3}\right), 1.73-2.00\left(\mathrm{CH}_{2}\right)$, $3.07-3.22\left(\mathrm{CH}_{3}\right), 3.35-3.50\left(\mathrm{CH}_{2}\right), 3.80-4.10\left(\mathrm{CH}_{2}\right)$, $4.40-4.55(\mathrm{CH}) ;{ }^{19} \mathrm{~F}$ NMR $\left(\mathrm{D}_{2} \mathrm{O}\right.$, ext. $\left.\mathrm{CF}_{3} \mathrm{CO}_{2} \mathrm{H}\right) \delta$ $-5.66(6 \mathrm{~F}),-43.02(4 \mathrm{~F}),-52.84(4 \mathrm{~F})$.

$\mathrm{R}_{\mathrm{F}}-(\mathrm{MHPTA})_{n}-\mathrm{R}_{\mathrm{F}}\left[\mathrm{R}_{\mathrm{F}}=\mathrm{CF}\left(\mathrm{CF}_{3}\right) \mathrm{OC}_{3} \mathrm{~F}_{7}\right.$; Run 3 in Table I]; IR $\left(\mathrm{cm}^{-1}\right) 3457(\mathrm{OH}), 1725(\mathrm{C}=\mathrm{O}), 1320$ $\left(\mathrm{CF}_{3}\right), 1244\left(\mathrm{CF}_{2}\right) ;{ }^{1} \mathrm{H}$ NMR $\left(\mathrm{D}_{2} \mathrm{O}\right) \delta 0.68-1.10\left(\mathrm{CH}_{3}\right)$, $1.60-2.10\left(\mathrm{CH}_{2}\right), 3.05-3.20\left(\mathrm{CH}_{3}\right), 3.35-3.50\left(\mathrm{CH}_{2}\right)$, $3.80-4.10\left(\mathrm{CH}_{2}\right), 4.43-4.55(\mathrm{CH}) ;{ }^{19} \mathrm{~F} \mathrm{NMR}\left(\mathrm{D}_{2} \mathrm{O}\right.$, ext. $\left.\mathrm{CF}_{3} \mathrm{CO}_{2} \mathrm{H}\right) \delta-5.63--6.93(16 \mathrm{~F}),-53.17(6 \mathrm{~F})$.

$\mathrm{R}_{\mathrm{F}}-(\mathrm{MHPTA})_{n}-\mathrm{R}_{\mathrm{F}}\left[\mathrm{R}_{\mathrm{F}}=\mathrm{CF}\left(\mathrm{CF}_{3}\right) \mathrm{OC}_{3} \mathrm{~F}_{7}\right.$; Run 4 in Table I]; IR ( $\left.\mathrm{cm}^{-1}\right) 3457(\mathrm{OH}), 1727(\mathrm{C}=\mathrm{O}), 1321$ $\left(\mathrm{CF}_{3}\right), 1241\left(\mathrm{CF}_{2}\right) ;{ }^{1} \mathrm{H}$ NMR $\left(\mathrm{D}_{2} \mathrm{O}\right) \delta 0.68-1.11\left(\mathrm{CH}_{3}\right)$, $1.61-2.15\left(\mathrm{CH}_{2}\right), 3.05-3.19\left(\mathrm{CH}_{3}\right), 3.31-3.54\left(\mathrm{CH}_{2}\right)$, $3.79-4.19\left(\mathrm{CH}_{2}\right), 4.43-4.55(\mathrm{CH}) ;{ }^{19} \mathrm{~F}$ NMR $\left(\mathrm{D}_{2} \mathrm{O}\right.$, ext. $\left.\mathrm{CF}_{3} \mathrm{CO}_{2} \mathrm{H}\right) \delta-5.63-6.93(16 \mathrm{~F}),-53.25(6 \mathrm{~F})$. $\mathrm{R}_{\mathrm{F}}-(\mathrm{MHPTA})_{n}-\mathrm{R}_{\mathrm{F}}\left[\mathrm{R}_{\mathrm{F}}=\mathrm{CF}\left(\mathrm{CF}_{3}\right) \mathrm{OC}_{3} \mathrm{~F}_{7} ; \mathrm{Run} 5\right.$ in Table I]; IR $\left(\mathrm{cm}^{-1}\right) 3455(\mathrm{OH}), 1727(\mathrm{C}=\mathrm{O}), 1310$ $\left(\mathrm{CF}_{3}\right), 1242\left(\mathrm{CF}_{2}\right) ;{ }^{1} \mathrm{H}$ NMR $\left(\mathrm{D}_{2} \mathrm{O}\right) \delta 0.68-1.12\left(\mathrm{CH}_{3}\right)$, $1.62-2.18\left(\mathrm{CH}_{2}\right), 3.08-3.22\left(\mathrm{CH}_{3}\right), 3.38-3.55\left(\mathrm{CH}_{2}\right)$, $3.80-4.14\left(\mathrm{CH}_{2}\right), 4.43-4.60(\mathrm{CH}) ;{ }^{19} \mathrm{~F}$ NMR $\left(\mathrm{D}_{2} \mathrm{O}\right.$, ext. $\left.\mathrm{CF}_{3} \mathrm{CO}_{2} \mathrm{H}\right) \delta-5.61--6.72(16 \mathrm{~F}),-53.04(6 \mathrm{~F})$.

$\mathrm{R}_{\mathrm{F}}-(\mathrm{MHPTA})_{n}-\mathrm{R}_{\mathrm{F}}\left[\mathrm{R}_{\mathrm{F}}=\mathrm{CF}\left(\mathrm{CF}_{3}\right) \mathrm{OC}_{3} \mathrm{~F}_{7} ;\right.$ Run 6 in Table I]; IR $\left(\mathrm{cm}^{-1}\right) 3464(\mathrm{OH}), 1724(\mathrm{C}=\mathrm{O}), 1320$ $\left(\mathrm{CF}_{3}\right), 1242\left(\mathrm{CF}_{2}\right) ;{ }^{1} \mathrm{H}$ NMR $\left(\mathrm{D}_{2} \mathrm{O}\right) \delta 0.60-1.13\left(\mathrm{CH}_{3}\right)$, $1.66-2.12\left(\mathrm{CH}_{2}\right), 3.06-3.33\left(\mathrm{CH}_{3}\right), 3.34-3.55\left(\mathrm{CH}_{2}\right)$, $3.80-4.12\left(\mathrm{CH}_{2}\right), 4.40-4.57(\mathrm{CH}) ;{ }^{19} \mathrm{~F}$ NMR $\left(\mathrm{D}_{2} \mathrm{O}\right.$, ext. $\left.\mathrm{CF}_{3} \mathrm{CO}_{2} \mathrm{H}\right) \delta-5.66-6.65(16 \mathrm{~F}),-53.61(6 \mathrm{~F})$.

$\mathrm{R}_{\mathrm{F}}-(\mathrm{MHPTA})_{n}-\mathrm{R}_{\mathrm{F}}\left[\mathrm{R}_{\mathrm{F}}=\mathrm{CF}\left(\mathrm{CF}_{3}\right) \mathrm{OC}_{3} \mathrm{~F}_{7} ;\right.$ Run 7 in Table I]; IR $\left(\mathrm{cm}^{-1}\right) 3438(\mathrm{OH}), 1724(\mathrm{C}=\mathrm{O}), 1319$ $\left(\mathrm{CF}_{3}\right), 1257\left(\mathrm{CF}_{2}\right) ;{ }^{1} \mathrm{H} \mathrm{NMR}\left(\mathrm{D}_{2} \mathrm{O}\right) \delta 0.50-1.20\left(\mathrm{CH}_{3}\right)$, $1.22-2.38\left(\mathrm{CH}_{2}\right), 3.38-3.37\left(\mathrm{CH}_{3}\right), 3.38-3.55\left(\mathrm{CH}_{2}\right)$, $3.80-4.22\left(\mathrm{CH}_{2}\right), 4.30-4.55(\mathrm{CH}) ;{ }^{19} \mathrm{~F}$ NMR $\left(\mathrm{D}_{2} \mathrm{O}\right.$, ext. $\left.\mathrm{CF}_{3} \mathrm{CO}_{2} \mathrm{H}\right) \delta-5.58--7.43(16 \mathrm{~F}),-53.59(6 \mathrm{~F})$.

$\mathrm{R}_{\mathrm{F}}-(\mathrm{MHPTA})_{n}-\mathrm{R}_{\mathrm{F}}\left[\mathrm{R}_{\mathrm{F}}=\mathrm{CF}\left(\mathrm{CF}_{3}\right) \mathrm{OCF}_{2} \mathrm{CF}\left(\mathrm{CF}_{3}\right)-\right.$ $\mathrm{OC}_{3} \mathrm{~F}_{7}$; Run 8 in Table I]; IR $\left(\mathrm{cm}^{-1}\right) 3462(\mathrm{OH}), 1715$ $(\mathrm{C}=\mathrm{O}), 1317\left(\mathrm{CF}_{3}\right), 1245\left(\mathrm{CF}_{2}\right) ;{ }^{1} \mathrm{H}$ NMR $\left(\mathrm{D}_{2} \mathrm{O}\right) \delta$ $0.60-1.10\left(\mathrm{CH}_{3}\right), 1.70-2.10\left(\mathrm{CH}_{2}\right), 3.05-3.24\left(\mathrm{CH}_{3}\right)$, $3.34-3.51\left(\mathrm{CH}_{2}\right), 3.80-4.11\left(\mathrm{CH}_{2}\right), 4.42-4.51(\mathrm{CH})$; ${ }^{19} \mathrm{~F}$ NMR $\left(\mathrm{D}_{2} \mathrm{O}\right.$, ext. $\left.\mathrm{CF}_{3} \mathrm{CO}_{2} \mathrm{H}\right) \delta-5.76--8.20$ (26F), $-53.04(6 \mathrm{~F}),-70.93(2 \mathrm{~F})$.

$\mathrm{R}_{\mathrm{F}}-(\mathrm{MHPTA})_{n}-\mathrm{R}_{\mathrm{F}}\left[\mathrm{R}_{\mathrm{F}}=\mathrm{CF}\left(\mathrm{CF}_{3}\right) \mathrm{OCF}_{2} \mathrm{CF}\left(\mathrm{CF}_{3}\right)-\right.$ $\mathrm{OC}_{3} \mathrm{~F}_{7}$; Run 9 in Table I]; IR $\left(\mathrm{cm}^{-1}\right) 3436(\mathrm{OH}), 1727$ $(\mathrm{C}=\mathrm{O}), 1323\left(\mathrm{CF}_{3}\right), 1257\left(\mathrm{CF}_{2}\right) ;{ }^{1} \mathrm{H}$ NMR $\left(\mathrm{D}_{2} \mathrm{O}\right) \delta$ $0.65-1.13\left(\mathrm{CH}_{3}\right), 1.70-2.20\left(\mathrm{CH}_{2}\right), 3.05-3.25\left(\mathrm{CH}_{3}\right)$, $3.35-3.53\left(\mathrm{CH}_{2}\right), 3.80-4.05\left(\mathrm{CH}_{2}\right), 4.32-4.55(\mathrm{CH})$; ${ }^{19} \mathrm{~F}$ NMR $\left(\mathrm{D}_{2} \mathrm{O}\right.$, ext. $\left.\mathrm{CF}_{3} \mathrm{CO}_{2} \mathrm{H}\right) \delta-5.76--7.84$ $(26 \mathrm{~F}),-53.01(6 \mathrm{~F}),-70.64(2 \mathrm{~F})$.

$\mathrm{R}_{\mathrm{F}}-(\mathrm{MHPTA})_{x}-\left(\mathrm{CH}_{2}-\mathrm{CHSiMe}_{3}\right)_{y}-\mathrm{R}_{\mathrm{F}}\left[\mathrm{R}_{\mathrm{F}}=\mathrm{CF}-\right.$ $\left(\mathrm{CF}_{3}\right) \mathrm{OC}_{3} \mathrm{~F}_{7}$; Run 17 in Table II]; IR $\left(\mathrm{cm}^{-1}\right) 3428(\mathrm{OH})$, $1724(\mathrm{C}=\mathrm{O}), 1257\left(\mathrm{CF}_{2}\right), 863\left(\mathrm{Si}-\mathrm{CH}_{3}\right) ;{ }^{1} \mathrm{H} \mathrm{NMR}\left(\mathrm{D}_{2} \mathrm{O}\right)$ $\delta-0.20-0\left(\mathrm{SiCH}_{3}\right), 0.65-1.10\left(\mathrm{CH}_{3}, \mathrm{CH}\right), 1.70-2.18$ $\left(\mathrm{CH}_{2}\right), 3.07-3.21\left(\mathrm{CH}_{3}\right), 3.35-3.48\left(\mathrm{CH}_{2}\right), 3.80-4.12$ $\left(\mathrm{CH}_{2}\right), 4.34-4.56(\mathrm{CH}) ;{ }^{19} \mathrm{~F}$ NMR $\left(\mathrm{D}_{2} \mathrm{O}\right.$, ext. $\mathrm{CF}_{3}-$ $\left.\mathrm{CO}_{2} \mathrm{H}\right) \delta-5.48--7.53(16 \mathrm{~F}),-53.01(6 \mathrm{~F})$.

$\mathrm{R}_{\mathrm{F}}-(\mathrm{MHPTA})_{x}-\left(\mathrm{CH}_{2}-\mathrm{CHSiMe}\right)_{y}-\mathrm{R}_{\mathrm{F}}\left[\mathrm{R}_{\mathrm{F}}=\mathrm{CF}-\right.$ $\left(\mathrm{CF}_{3}\right) \mathrm{OCF}_{2} \mathrm{CF}\left(\mathrm{CF}_{3}\right) \mathrm{OC}_{3} \mathrm{~F}_{7}$; Run 18 in Table II]; IR $\left(\mathrm{cm}^{-1}\right) 3467(\mathrm{OH}), 1730(\mathrm{C}=\mathrm{O}), 1323\left(\mathrm{CF}_{3}\right), 1245\left(\mathrm{CF}_{2}\right)$, $860\left(\mathrm{Si}-\mathrm{CH}_{3}\right) ;{ }^{1} \mathrm{H}$ NMR $\left(\mathrm{D}_{2} \mathrm{O}\right) \delta-0.30-0\left(\mathrm{SiCH}_{3}\right)$, $0.65-1.15\left(\mathrm{CH}_{3}, \mathrm{CH}\right), 1.70-2.20\left(\mathrm{CH}_{2}\right), 3.07-3.25$ $\left(\mathrm{CH}_{3}\right), 3.35-3.60\left(\mathrm{CH}_{2}\right), 3.80-4.13\left(\mathrm{CH}_{2}\right), 4.40-4.60$ $(\mathrm{CH}) ;{ }^{19} \mathrm{~F} \mathrm{NMR}\left(\mathrm{D}_{2} \mathrm{O}\right.$, ext. $\left.\mathrm{CF}_{3} \mathrm{CO}_{2} \mathrm{H}\right) \delta-5.56--7.92$ $(26 \mathrm{~F}),-53.04(6 \mathrm{~F}),-70.50(2 \mathrm{~F})$.

Similarly, a series of fluoroalkylated end-capped HOPPA oligomers was prepared by oligomerization with fluoroalkanoyl peroxides, and exhibited the following spectral characteristics:

$\mathrm{R}_{\mathrm{F}}-(\mathrm{HOPPA})_{n}-\mathrm{R}_{\mathrm{F}}\left[\mathrm{R}_{\mathrm{F}}=\mathrm{C}_{3} \mathrm{~F}_{7}\right.$; Run 10 in Table I]; IR $\left(\mathrm{cm}^{-1}\right) 3471(\mathrm{OH}), 3164(\mathrm{NH}), 1736,1648(\mathrm{C}=\mathrm{O}), 1313$ $\left(\mathrm{CF}_{3}\right), 1261\left(\mathrm{CF}_{2}\right) ;{ }^{1} \mathrm{H} \mathrm{NMR}\left(\mathrm{D}_{2} \mathrm{O}\right) \delta 0.90-2.40\left(\mathrm{CH}_{2}\right.$, $\mathrm{CH}), 5.10-5.57(\mathrm{CH}) ;{ }^{19} \mathrm{~F}$ NMR $\left(\mathrm{D}_{2} \mathrm{O}\right.$, ext. $\left.\mathrm{CF}_{3} \mathrm{CO}_{2} \mathrm{H}\right)$ $\delta-5.63,-7.77(6 \mathrm{~F}),-43.17,-45.48(4 \mathrm{~F}),-53.74$, $54.65(4 \mathrm{~F})$.

$\mathrm{R}_{\mathrm{F}}-(\mathrm{HOPPA})_{n}-\mathrm{R}_{\mathrm{F}}\left[\mathrm{R}_{\mathrm{F}}=\mathrm{CF}\left(\mathrm{CF}_{3}\right) \mathrm{OC}_{3} \mathrm{~F}_{7}\right.$; Run 11 in Table I]; IR $\left(\mathrm{cm}^{-1}\right) 3465(\mathrm{OH}), 3187(\mathrm{NH}), 1745,1658$ $(\mathrm{C}=\mathrm{O}), 1336\left(\mathrm{CF}_{3}\right), 1257\left(\mathrm{CF}_{2}\right) ;{ }^{1} \mathrm{H} \mathrm{NMR}\left(\mathrm{D}_{2} \mathrm{O}\right) \delta$ $0.98-2.48\left(\mathrm{CH}_{2}, \mathrm{CH}\right), 5.09-5.62(\mathrm{CH}) ;{ }^{19} \mathrm{~F}$ NMR $\left(\mathrm{D}_{2} \mathrm{O}\right.$, ext. $\left.\mathrm{CF}_{3} \mathrm{CO}_{2} \mathrm{H}\right) \delta-6.96--7.76(16 \mathrm{~F}),-54.15$ 
(6F).

$\mathrm{R}_{\mathrm{F}}-(\mathrm{HOPPA})_{n}-\mathrm{R}_{\mathrm{F}}\left[\mathrm{R}_{\mathrm{F}}=\mathrm{CF}\left(\mathrm{CF}_{3}\right) \mathrm{OC}_{3} \mathrm{~F}_{7} ;\right.$ Run 12 in Table I]; IR $\left(\mathrm{cm}^{-1}\right) 3457(\mathrm{OH}), 3195(\mathrm{NH}), 1747,1662$ $(\mathrm{C}=\mathrm{O}), 1334\left(\mathrm{CF}_{3}\right), 1226\left(\mathrm{CF}_{2}\right) ;{ }^{1} \mathrm{H}$ NMR $\left(\mathrm{D}_{2} \mathrm{O}\right) \delta$ $0.80-2.51\left(\mathrm{CH}_{2}, \mathrm{CH}\right), 5.15-5.81(\mathrm{CH}) ;{ }^{19} \mathrm{~F}$ NMR $\left(\mathrm{D}_{2} \mathrm{O}\right.$, ext. $\left.\mathrm{CF}_{3} \mathrm{CO}_{2} \mathrm{H}\right) \delta-5.76--7.76(16 \mathrm{~F}),-54.21$ (6F).

$\mathrm{R}_{\mathrm{F}}-(\mathrm{HOPPA})_{n}-\mathrm{R}_{\mathrm{F}}\left[\mathrm{R}_{\mathrm{F}}=\mathrm{CF}\left(\mathrm{CF}_{3}\right) \mathrm{OC}_{3} \mathrm{~F}_{7}\right.$; Run 13 in Table I]; IR $\left(\mathrm{cm}^{-1}\right) 3457(\mathrm{OH}), 3181(\mathrm{NH}), 1743,1653$ $(\mathrm{C}=\mathrm{O}), 1255\left(\mathrm{CF}_{2}\right) ;{ }^{1} \mathrm{H}$ NMR $\left(\mathrm{D}_{2} \mathrm{O}\right) \delta 0.96-2.49\left(\mathrm{CH}_{2}\right.$, $\mathrm{CH}), 5.01-5.80(\mathrm{CH}) ;{ }^{19} \mathrm{~F} \mathrm{NMR}\left(\mathrm{D}_{2} \mathrm{O}\right.$, ext. $\left.\mathrm{CF}_{3} \mathrm{CO}_{2} \mathrm{H}\right)$ $\delta-4.43--8.62(16 \mathrm{~F}),-55.53(6 \mathrm{~F})$.

$\mathrm{R}_{\mathrm{F}}-(\mathrm{HOPPA})_{n}-\mathrm{R}_{\mathrm{F}}\left[\mathrm{R}_{\mathrm{F}}=\mathrm{CF}\left(\mathrm{CF}_{3}\right) \mathrm{OCF}_{2} \mathrm{CF}\left(\mathrm{CF}_{3}\right)-\right.$ $\mathrm{OC}_{3} \mathrm{~F}_{7}$; Run 14 in Table I]; IR $\left(\mathrm{cm}^{-1}\right) 3465(\mathrm{OH}), 3178$ $(\mathrm{NH}), 1753,1670(\mathrm{C}=\mathrm{O}), 1338\left(\mathrm{CF}_{3}\right), 1240\left(\mathrm{CF}_{2}\right) ;{ }^{1} \mathrm{H}$ $\operatorname{NMR}\left(\mathrm{D}_{2} \mathrm{O}\right) \delta 0.93-2.48\left(\mathrm{CH}_{2}, \mathrm{CH}\right), 5.00-5.63(\mathrm{CH})$; ${ }^{19} \mathrm{~F}$ NMR $\left(\mathrm{D}_{2} \mathrm{O}\right.$, ext. $\left.\mathrm{CF}_{3} \mathrm{CO}_{2} \mathrm{H}\right) \delta-5.76--7.06$ (26F), - 53.77- - 54.68 (6F), - $74.98(2 \mathrm{~F})$.

$\mathrm{R}_{\mathrm{F}}-(\mathrm{HOPPA})_{n}-\mathrm{R}_{\mathrm{F}}\left[\mathrm{R}_{\mathrm{F}}=\mathrm{CF}\left(\mathrm{CF}_{3}\right) \mathrm{OCF}_{2} \mathrm{CF}\left(\mathrm{CF}_{3}\right)-\right.$ $\mathrm{OC}_{3} \mathrm{~F}_{7}$; Run 15 in Table I]; IR $\left(\mathrm{cm}^{-1}\right) 3432(\mathrm{OH}), 3195$ $(\mathrm{NH}), 1745,1660(\mathrm{C}=\mathrm{O}), 1335\left(\mathrm{CF}_{3}\right), 1249\left(\mathrm{CF}_{2}\right)$; NMR spectra were not measured due to gelling (highly viscoelastic) of the sample.

$\mathrm{R}_{\mathrm{F}}$-(HOPPA $)_{n}-\mathrm{R}_{\mathrm{F}}\left[\mathrm{R}_{\mathrm{F}}=\mathrm{CF}\left(\mathrm{CF}_{3}\right) \mathrm{OCF}_{2} \mathrm{CF}\left(\mathrm{CF}_{3}\right)-\right.$ $\mathrm{OC}_{3} \mathrm{~F}_{7}$; Run 16 in Table I]; IR $\left(\mathrm{cm}^{-1}\right) 3465(\mathrm{OH}), 3178$ $(\mathrm{NH}), 1743,1662(\mathrm{C}=\mathrm{O}), 1338\left(\mathrm{CF}_{3}\right), 1240\left(\mathrm{CF}_{2}\right)$; NMR spectra were not measured due to gelling (highly viscoelastic) of the sample.

$\mathrm{R}_{\mathrm{F}}-(\mathrm{HOPPA})_{x}-\left(\mathrm{CH}_{2} \mathrm{CMeCO}_{2} \mathrm{Me}\right)_{y}-\mathrm{R}_{\mathrm{F}}\left[\mathrm{R}_{\mathrm{F}}=\mathrm{CF}-\right.$ $\left(\mathrm{CF}_{3}\right) \mathrm{OC}_{3} \mathrm{~F}_{7}$; Run 19 in Table II]; IR $\left(\mathrm{cm}^{-1}\right) 3463(\mathrm{OH})$, $3184(\mathrm{NH}), 1747,1662(\mathrm{C}=\mathrm{O}), 1325\left(\mathrm{CF}_{3}\right), 1240\left(\mathrm{CF}_{2}\right)$; ${ }^{1} \mathrm{H}$ NMR $\left(\mathrm{D}_{2} \mathrm{O}\right) \delta 0.88-2.89\left(\mathrm{CH}_{2}, \mathrm{CH}_{3}, \mathrm{CH}\right), 3.57-$ $3.67\left(\mathrm{CH}_{3}\right), 5.15-5.50(\mathrm{CH}) ;{ }^{19} \mathrm{~F}$ NMR $\left(\mathrm{D}_{2} \mathrm{O}\right.$, ext. $\left.\mathrm{CF}_{3} \mathrm{CO}_{2} \mathrm{H}\right) \delta-5.82--7.66(16 \mathrm{~F}),-54.23(6 \mathrm{~F})$.

$\mathrm{R}_{\mathrm{F}}-(\mathrm{HOPPA})_{x}-\left(\mathrm{CH}_{2} \mathrm{CMeCO}_{2} \mathrm{Me}\right)_{y}-\mathrm{R}_{\mathrm{F}}\left[\mathrm{R}_{\mathrm{F}}=\mathrm{CF}-\right.$ $\left(\mathrm{CF}_{3}\right) \mathrm{OC}_{3} \mathrm{~F}_{7}$; Run 20 in Table II]; IR $\left(\mathrm{cm}^{-1}\right) 3463(\mathrm{OH})$, $3180(\mathrm{NH}), 1738,1676(\mathrm{C}=\mathrm{O}), 1327\left(\mathrm{CF}_{3}\right), 1242\left(\mathrm{CF}_{2}\right)$; ${ }^{1} \mathrm{H}$ NMR $\left(\mathrm{D}_{2} \mathrm{O}\right) \delta 0.99-2.70\left(\mathrm{CH}_{2}, \mathrm{CH}_{3}, \mathrm{CH}\right), 3.47-$ $3.63\left(\mathrm{CH}_{3}\right), 5.10-5.64(\mathrm{CH}) ;{ }^{19} \mathrm{~F}$ NMR $\left(\mathrm{D}_{2} \mathrm{O}\right.$, ext. $\left.\mathrm{CF}_{3} \mathrm{CO}_{2} \mathrm{H}\right) \delta-5.76--6.88(16 \mathrm{~F}),-54.36(6 \mathrm{~F})$.

\section{Viscosity Measurements}

Viscosity of aqueous solution of fluoroalkylated end-capped MHPTA oligomers was measured at $30^{\circ} \mathrm{C}$ using a falling-sphere viscometer (Haake Viscometer D1-G).

\section{A Typical Procedure for Gelation Test}

The procedure for studying the gel-formation ability was essentially that of Hanabusa et al. ${ }^{15}$ Briefly, weighed fluoroalkylated end-capped oligomer was mixed with water or organic fluid in a tube. The mixture was treated under ultrasonic conditions until the solid was dissolved. The resulting solution was kept at $30^{\circ} \mathrm{C}$ for $1 \mathrm{~h}$, and gelation was checked visually. The gel was stable and the tube could be inverted without changing the shape of the gel.

\section{Metal Ions Binding or Releasing by Fluoroalkylated End-Capped Oligomer Hydrogels}

Fluoroalkylated end-capped oligomer hydrogels were swollen with water in a measuring flask. After the addition of the required amount of aqueous metal ion

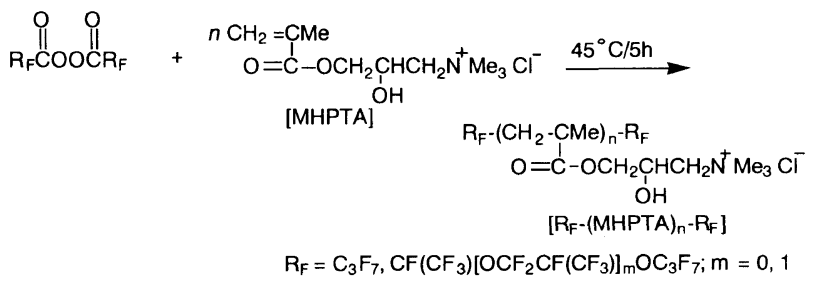

Scheme 1.

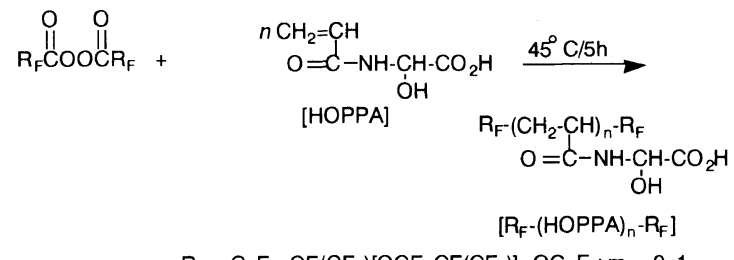

$\mathrm{R}_{\mathrm{F}}=\mathrm{C}_{3} \mathrm{~F}_{7}, \mathrm{CF}\left(\mathrm{CF}_{3}\right)\left[\mathrm{OCF}_{2} \mathrm{CF}\left(\mathrm{CF}_{3}\right)\right]_{\mathrm{m}} \mathrm{OC}_{3} \mathrm{~F}_{7} ; \mathrm{m}=0,1$

Scheme 2.

solution into the flask, the flask was allowed to stand for 1 day at $25^{\circ} \mathrm{C}$. The metal-ion concentration of supernatant liquid after incubation was spectrophotometrically determined.

Metal ion was released from the metal binding to the hydrogel into water for 1 day at $25^{\circ} \mathrm{C}$, and its concentration in the supernatant liquid was determined spectrophotometrically.

\section{RESULTS AND DISCUSSION}

Reactions of fluoroalkanoyl peroxides with methacrylate monomer containing trimethylammonium and hydroxy segments (MHPTA) were found to proceed under very mild conditions to afford fluoroalkylated end-capped 3-methacryloxy-2-hydroxypropyltrimethylammonium chloride oligomers as shown in Scheme 1.

We prepared a series of fluoroalkylated end-capped oligomers containing hydroxy and carboxy segments by reactions of fluoroalkanoyl peroxides with 2-hydroxy2 -[(1-oxoprop-2-enyl)amino]acetic acid [HOPPA] as shown in Scheme 2.

The results of the reactions of fluoroalkanoyl peroxides with MHPTA or HOPPA are summarized in Table I.

We also prepared fluoroalkylated end-capped MHPTA or HOPPA co-oligomers using co-monomers such as trimethylvinylsilane and methyl methacrylate as shown in Scheme 3 and Table II.

As Tables I and II show, perfluoropropylated and some perfluoro-oxaalkylated MHPTA or HOPPA homo- and co-oligomers were obtained in excellent to moderate isolated yields under very mild conditions. The yields of perfluoro-1-methyl-2-oxapentylated MHPTA oligomers (No. 3-7) and perfluoro-1-methyl-2-oxapentylated HOPPA-methyl methacrylate co-oligomers (No. 19, 20) are not dependent upon the molar ratios of monomers and peroxides, but possibly on homoand co-oligomerization of MHPTA (or HOPPA) with peroxides that are heterogeneous systems including water.

In these fluoroalkylated end-capped oligomers, perfluoropropylated and perfluoro-1-methyl-2-oxapentylated MHPTA oligomers (No. 1, 2, 3 in Table I) were soluble in water, and we were able to measure molecular weights 
by GPC (gel permeation chromatography) analysis calibrated with standard poly(ethylene glycol) using $30 \%$ acetonitrile solution containing $0.5 \mathrm{M}$ acetic acid and $0.5 \mathrm{M}$ sodium acetate as the eluent $\left[\bar{M}_{n}\left(\bar{M}_{w} / \bar{M}_{n}\right)=2630\right.$ (3.02) (No. 1); 2060 (3.86) (No. 2); 6760 (11.0) (No. 3)]. In perfluoro-1-methyl-2-oxapentylated MHPTA oligomers, the oligomers with greater molar ratios of MHPTA

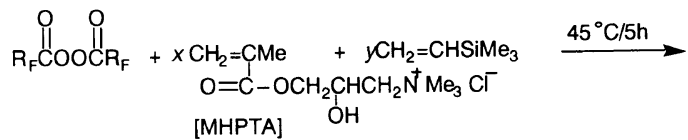

$$
\begin{aligned}
& \mathrm{R}_{\mathrm{F}}-\left(\mathrm{CH}_{2}-\mathrm{CMe}\right)_{x}-\left(\mathrm{CH}_{2}-\mathrm{CHSiMe}\right)_{3}-\mathrm{R}_{\mathrm{F}} \\
& \mathrm{O}=\mathrm{C}-\mathrm{OCH}_{2} \mathrm{CHCH}_{2} \mathrm{~N}^{\top} \mathrm{Me}_{3} \mathrm{Cr} \\
& {\left[\mathrm{R}_{\mathrm{F}}-(\mathrm{MHPTA})_{x}-\left(\mathrm{CH}_{2} \mathrm{CHSiMe}\right)_{3}-\mathrm{R}_{\mathrm{F}}\right]} \\
& \stackrel{\mathrm{O}}{\mathrm{R}_{\mathrm{F}} \mathrm{COOCR}_{\mathrm{F}}}+\underset{\substack{\mathrm{O}=\mathrm{C}-\mathrm{NH}-\underset{\mathrm{CH}}{\mathrm{C}}-\mathrm{CO}_{2} \mathrm{H} \\
\text { [HOPPA] } \mathrm{OH}}}{\stackrel{\mathrm{CH}}{\mathrm{O}}=\mathrm{CH}} \\
& \mathrm{R}_{\mathrm{F}}-\left(\mathrm{CH}_{2}-\mathrm{CH}\right)_{x}-\left(\mathrm{CH}_{2}-\mathrm{CMeCO}_{2} \mathrm{Me}\right)_{y}-\mathrm{R}_{\mathrm{F}} \\
& \mathrm{O}=\mathrm{C}-\mathrm{NH}-\mathrm{CH}-\mathrm{CO}_{2} \mathrm{H} \\
& \mathrm{OH} \\
& {\left[\mathrm{R}_{\mathrm{F}}-(\mathrm{HOPPA})_{x}-\left(\mathrm{CH}_{2}-\mathrm{CMeCO}_{2} \mathrm{Me}\right)_{y}-\mathrm{R}_{\mathrm{F}}\right.}
\end{aligned}
$$

$\mathrm{R}_{\mathrm{F}}=\mathrm{CF}\left(\mathrm{CF}_{3}\right)\left[\mathrm{OCF}_{2} \mathrm{CF}\left(\mathrm{CF}_{3}\right)\right]_{\mathrm{m}} \mathrm{OC}_{3} \mathrm{~F}_{7} ; \mathrm{m}=0,1$

Scheme 3. in MHPTA/peroxide (No. 4-9) became "gel-like" or formed gels with water and polar organic solvents such as methanol, ethanol, dimethylformamide and dimethyl sulfoxide, and failed to measure their molecular weights owing to the highly viscoelastic fluids or the gel formation. Both perfluoropropylated and perfluoro-oxaalkylated HOPPA oligomers were found to cause gelation with water, dimethylformamide and dimethyl sulfoxide under the non-crosslinked conditions, and we could not measure the molecular weight of all oligomers by GPC. It is important to note that corresponding nonfluorinated MHPTA or HOPPA oligomer [-(MHPTA $)_{n}-$ or -(HOPPA $)_{n-}$ ] forms no gels with these solvents, and these non-fluorinated oligomers are completely soluble in these solvents.

Perfluoro-1-methyl-2-oxapentylated end-capped MHPTA-trimethylvinylsilane co-oligomer (No. 17) in Table II formed highly viscoelastic fluids (gel-like) in water and polar organic solvents such as $\mathrm{MeOH}$, EtOH, $N, N$-dimethylformamide (DMF), and dimethylsulfoxide (DMSO), and the longer perfluoro-oxaalkylated cooligomer (No. 18) in Table II was shown to cause gela-

\begin{tabular}{|c|c|c|c|c|c|}
\hline \multirow{2}{*}{ Run } & \multirow{2}{*}{$\frac{\mathrm{R}_{\mathrm{F}} \text { in peroxide }}{\mathrm{mmol}}$} & \multirow{2}{*}{$\frac{\text { MHPTA (or HOPPA) }}{\text { mmol }}$} & \multirow{2}{*}{$\frac{\text { Co-monmer }}{\mathrm{mmol}}$} & \multicolumn{2}{|c|}{ Product } \\
\hline & & & & Yield $/ \%^{\mathbf{a}}$ & $x: y^{\mathrm{b}}$ \\
\hline & & MHPTA & $\mathrm{CH}_{2}=\mathrm{CHSiMe}_{3}$ & \multicolumn{2}{|c|}{$\mathrm{R}_{\mathrm{F}}-(\mathrm{MHPTA})_{x}-\left(\mathrm{CH}_{2}-\mathrm{CHSiMe}\right)_{y}-\mathrm{R}_{\mathrm{F}}$} \\
\hline 17 & $\mathrm{CF}\left(\mathrm{CF}_{3}\right) \mathrm{OC}_{3} \mathrm{~F}_{7}$ & 17 & 8 & 31 & $96: 4$ \\
\hline 18 & $\mathrm{CF}\left(\mathrm{CF}_{3}\right) \mathrm{OCF}_{2} \mathrm{CF}\left(\mathrm{CF}_{3}\right) \mathrm{OC}_{3} \mathrm{~F}_{7}$ (4) & 16 & 8 & 27 & $95: 5$ \\
\hline 19 & $\mathrm{CF}\left(\mathrm{CF}_{3}\right) \mathrm{OC}_{3} \mathrm{~F}_{7}(4)$ & 8 & 8 & 45 & $70: 30$ \\
\hline 20 & $\mathrm{CF}\left(\mathrm{CF}_{3}\right) \mathrm{OC}_{3} \mathrm{~F}_{7}(4)$ & 13 & 84 & 9 & $30: 70$ \\
\hline
\end{tabular}
tion in these solvents. Fluoroalkylated HOPPA-methyl methacrylate co-oligomer (No. 19) in Table II caused gelation in water, DMF, and DMSO. In contrast, flu-

Table I. Reactions of fluoroalkanoyl peroxides with MHPTA (or HOPPA)

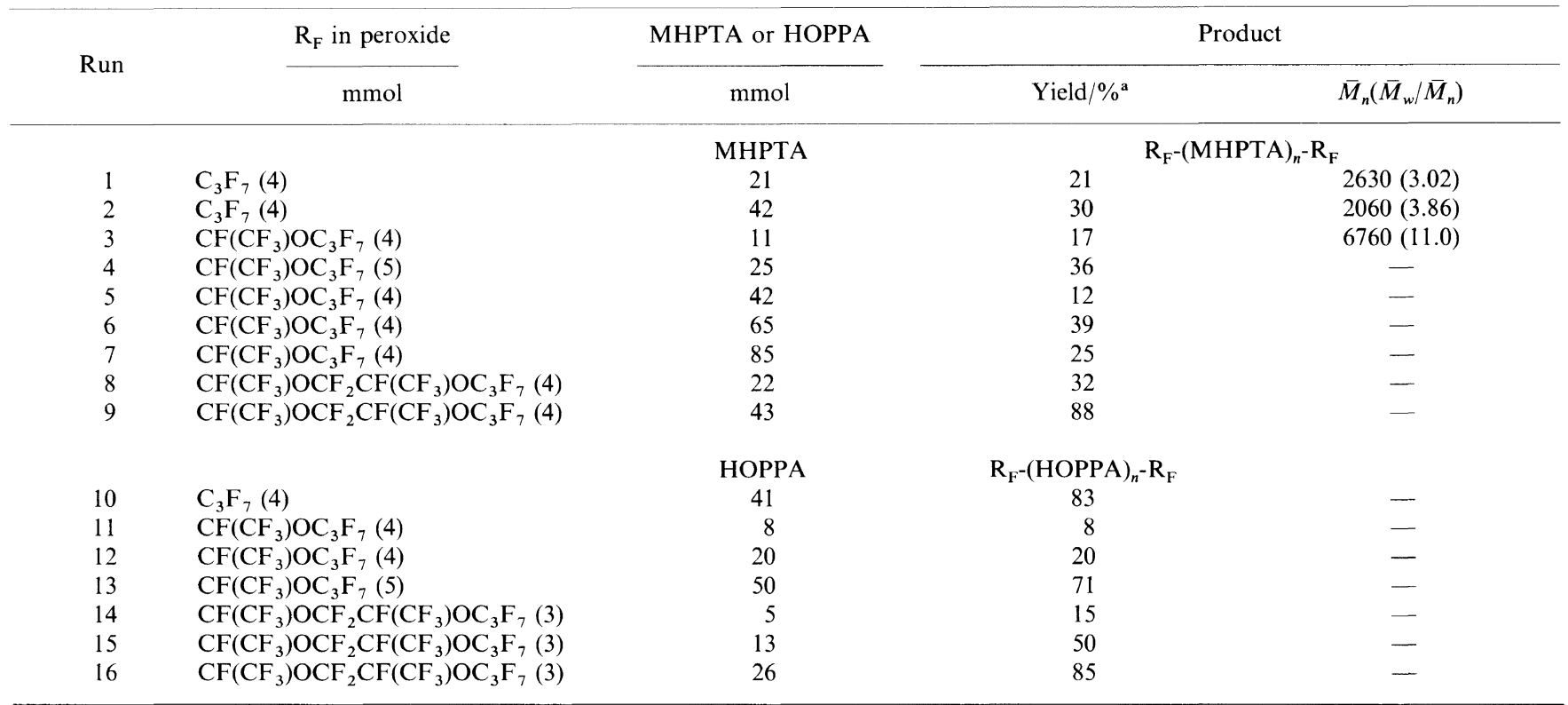

${ }^{a}$ Yields based on starting materials [MHPTA (or HOPPA)] and decarboxylated peroxide unit $\left(\mathrm{R}_{\mathrm{F}}-\mathrm{R}_{\mathrm{F}}\right)$.

Table II. Reactions of fluoroalkanoyl peroxides with MHPTA (or HOPPA) and trimethylvinylsilane (or methyl methacrylate)

a Yields based on starting materials [MHPTA (or HOPPA), trimethylvinylsilane (or methyl methacrylate)] and the decarboxylated peroxide unit $\left(R_{F}-R_{F}\right)$. ${ }^{b}$ Co-oligomerization ratio determined by ${ }^{1} \mathrm{H} N M R$. 
oroalkylated HOPPA co-oligomer (No. 20) was insoluble in water, and caused gelation only in organic polar solvents such as DMF and DMSO. This would depend upon higher oleophilic property than the corresponding co-oligomer (No. 19).

To study the unique gelation of these fluorinated oligomers under non-crosslinked conditions, we measured the viscosity of aqueous solutions of gel-like perfluoro-1-methyl-2-oxapentylated MHPTA oligomer (No. 5) and soluble perfluoropropylated MHPTA oligomer (No. 2) at $30^{\circ} \mathrm{C}$. The results are shown in Figure 1.

As shown in Figure 1, the viscosity of perfluoropropylated MHPTA oligomer (No. 2) did not increase remarkably with concentrations, and gels or highly viscoelastic fluids did not form even under a higher concentration $\left(300 \mathrm{~g} \mathrm{dm}^{-3}\right)$. In contrast, the viscosity of

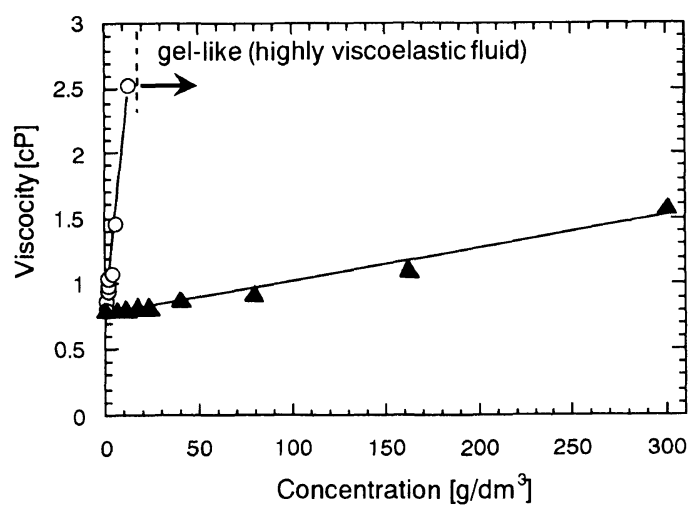

Figure 1. Effects of concentration on viscosity of $R_{F}-(M H P T A)_{n}-R_{F}$ at $30^{\circ} \mathrm{C} . \Delta, \mathrm{R}_{\mathrm{F}}=\mathrm{C}_{3} \mathrm{~F}_{7}\left(\right.$ No. 2 in Table I); $\mathrm{O}, \mathrm{R}_{\mathrm{F}}=\mathrm{CF}\left(\mathrm{CF}_{3}\right) \mathrm{OC}_{3} \mathrm{~F}_{7}$ (No. 5 in Table I). perfluoro-1-methyl-2-oxapentylated MHPTA oligomer (No. 5) increased remarkably with concentration, and we were not able to measure viscosity owing to the formation of highly viscoelastic fluids (gel-like) above $c a$. $10 \mathrm{~g} \mathrm{dm}^{-3}$. These results suggest that interactions of the aggregation of longer fluoroalkyl segments become stronger to establish highly viscous gel-like fluid or a physical gel network. For low-molecular compounds, Hanabusa et al. reported a similar remarkable rise of viscosity of organic solvents containing trialkyl-1,3,5benzenetricarboxyamides to that of aqueous solutions of our present fluorinated oligomer in Figure 1 and intermolecular hydrogen bonding as the main driving force for the gelation. ${ }^{16}$

The gelation ability of fluoroalkylated end-capped MHPTA or HOPPA homo- and co-oligomers were also studied by measuring minimum concentrations $\left(C_{\mathrm{min}}\right)$ of these oligomers necessary for gelation in water, $\mathrm{MeOH}$, and DMSO at $30^{\circ} \mathrm{C}$ according to the method reported of Hanabusa et al., ${ }^{15}$ and the results are summarized in Table III.

As shown in Table III, $C_{\min }$ s of a series of fluoroalkylated homo- and co-oligomers necessary to gel one liter of water, $\mathrm{MeOH}$, and DMSO was $7-330 \mathrm{~g} \mathrm{dm}^{-3}$, and oligomers with greater molar ratios of MHPTA [No. 1, 2; No. 3-7; No. 8, 9] or HOPPA [No. 11-13; No. 14 -16] in MHPTA (or HOPPA)/peroxide or longer perfluoro-oxaalkylated oligomers exhibited higher gelling ability. These results strongly suggest that the main driving force for gelation is the synergistical interactions with the aggregation of fluoroalkyl units in oligomers and intermolecular hydrogen bonding between hydroxy segments or carboxy segments including the interactions

Table III. Minimum gel concentration $\left(C_{\min }\right)$ of fluoroalkylated MHPTA (or HOPPA) homo- and co-oligomers (in $\mathrm{g}$ per $\mathrm{dm}^{3}$ solvent) for gelation at $30^{\circ} \mathrm{C}$

\begin{tabular}{|c|c|c|c|c|}
\hline \multirow{2}{*}{ Run $^{\mathbf{a}}$} & \multirow{2}{*}{$\mathrm{R}_{\mathrm{F}}$ in oligomer } & \multicolumn{3}{|c|}{$C_{\min }^{\mathrm{b}}$} \\
\hline & & $\mathrm{H}_{2} \mathrm{O}$ & $\mathrm{MeOH}$ & DMSO \\
\hline \multicolumn{5}{|c|}{$\mathrm{R}_{\mathrm{F}^{-}}(\mathrm{MHPTA})_{n}-\mathrm{R}_{\mathrm{F}}$} \\
\hline 1 & $\mathrm{C}_{3} \mathrm{~F}_{7}$ & Soln & Soln & Soln \\
\hline 2 & $\mathrm{C}_{3} \mathrm{~F}_{7}$ & Soln & Soln & Soln \\
\hline 3 & $\mathrm{CF}\left(\mathrm{CF}_{3}\right) \mathrm{OC}_{3} \mathrm{~F}_{7}$ & Soln & Soln & Soln \\
\hline 4 & $\mathrm{CF}\left(\mathrm{CF}_{3}\right) \mathrm{OC}_{3} \mathrm{~F}_{7}$ & Gel-like & Gel-like & Gel-like \\
\hline 5 & $\mathrm{CF}\left(\mathrm{CF}_{3}\right) \mathrm{OC}_{3} \mathrm{~F}_{7}$ & Gel-like & Gel-like & Gel-like \\
\hline 6 & $\mathrm{CF}\left(\mathrm{CF}_{3}\right) \mathrm{OC}_{3} \mathrm{~F}_{7}$ & 102 & 121 & 191 \\
\hline 7 & $\mathrm{CF}\left(\mathrm{CF}_{3}\right) \mathrm{OC}_{3} \mathrm{~F}_{7}$ & 7 & 15 & 30 \\
\hline 8 & $\mathrm{CF}\left(\mathrm{CF}_{3}\right) \mathrm{OCF}_{2} \mathrm{CF}\left(\mathrm{CF}_{3}\right) \mathrm{OC}_{3} \mathrm{~F}_{7}$ & 330 & Gel-like & Gel-like \\
\hline 9 & $\mathrm{CF}\left(\mathrm{CF}_{3}\right) \mathrm{OCF}_{2} \mathrm{CF}\left(\mathrm{CF}_{3}\right) \mathrm{OC}_{3} \mathrm{~F}_{7}$ & 51 & 84 & 102 \\
\hline \multicolumn{5}{|c|}{$\mathrm{R}_{\mathrm{F}}-(\mathrm{HOPPA})_{n}-\mathrm{R}_{\mathrm{F}}$} \\
\hline 10 & $\mathrm{C}_{3} \mathrm{~F}_{7}$ & 88 & - & 145 \\
\hline 11 & $\mathrm{CF}\left(\mathrm{CF}_{3}\right) \mathrm{OC}_{3} \mathrm{~F}_{7}$ & 100 & - & 147 \\
\hline 13 & $\mathrm{CF}\left(\mathrm{CF}_{3}\right) \mathrm{OC}_{3} \mathrm{~F}_{7}$ & 91 & - & - \\
\hline 14 & $\mathrm{CF}\left(\mathrm{CF}_{3}\right) \mathrm{OCF}_{2} \mathrm{CF}\left(\mathrm{CF}_{3}\right) \mathrm{OC}_{3} \mathrm{~F}_{7}$ & 94 & - & 151 \\
\hline 15 & $\mathrm{CF}\left(\mathrm{CF}_{3}\right) \mathrm{OCF}_{2} \mathrm{CF}\left(\mathrm{CF}_{3}\right) \mathrm{OC}_{3} \mathrm{~F}_{7}$ & 87 & - & - \\
\hline 16 & $\mathrm{CF}\left(\mathrm{CF}_{3}\right) \mathrm{OCF}_{2} \mathrm{CF}\left(\mathrm{CF}_{3}\right) \mathrm{OC}_{3} \mathrm{~F}_{7}$ & 53 & 一 & 119 \\
\hline \multicolumn{5}{|c|}{$\mathrm{R}_{\mathrm{F}}-(\mathrm{MHPTA})_{x}-\left(\mathrm{CH}_{2} \mathrm{CHSiMe}_{3}\right)_{y}-\mathrm{R}_{\mathrm{F}}$} \\
\hline 17 & $\mathrm{CF}\left(\mathrm{CF}_{3}\right) \mathrm{OC}_{3} \mathrm{~F}_{7}$ & Gel-like & Gel-like & Gel-like \\
\hline 18 & $\mathrm{CF}\left(\mathrm{CF}_{3}\right) \mathrm{OCF}_{2} \mathrm{CF}\left(\mathrm{CF}_{3}\right) \mathrm{OC}_{3} \mathrm{~F}_{7}$ & 255 & 330 & Gel-like \\
\hline \multicolumn{5}{|c|}{$\mathrm{R}_{\mathrm{F}^{-}}(\mathrm{HOPPA})_{x}-\left(\mathrm{CH}_{2} \mathrm{CMeCO}_{2} \mathrm{Me}\right)_{y}-\mathrm{R}_{\mathrm{F}}$} \\
\hline 19 & $\mathrm{CF}\left(\mathrm{CF}_{3}\right) \mathrm{OC}_{3} \mathrm{~F}_{7}$ & 89 & - & 262 \\
\hline 20 & $\mathrm{CF}\left(\mathrm{CF}_{3}\right) \mathrm{OCF}_{2} \mathrm{CF}\left(\mathrm{CF}_{3}\right) \mathrm{OC}_{3} \mathrm{~F}_{7}$ & 一 & - & 125 \\
\hline
\end{tabular}

${ }^{\mathrm{a}}$ Each different from those in Tables I and II. ${ }^{\mathrm{b}}$ Gel-like= highly viscoelastic fluid. 


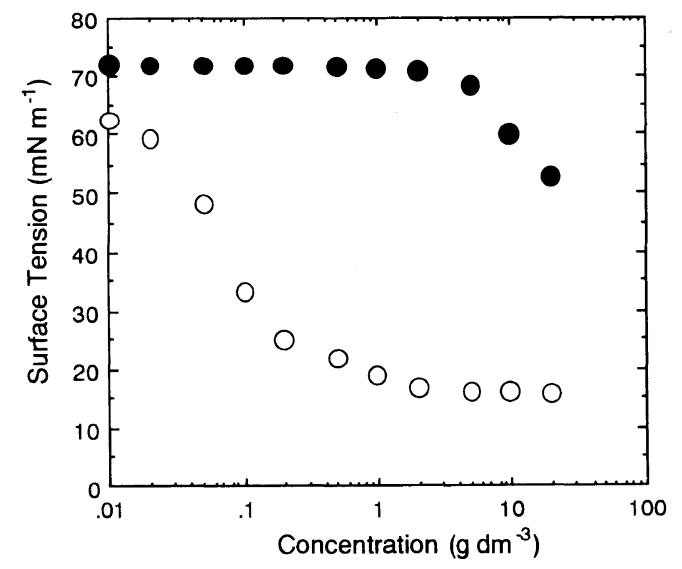

Figure 2. Surface tension of aqueous solutions of $R_{F}-(\text { MHPTA })_{n}-R_{F}$ (O: No. 5) and $-(\text { MHPTA })_{n}-(\mathbf{O})$ at $30^{\circ} \mathrm{C}$.

of hydroxy $(-\mathrm{OH})$ and trimethylammonium $\left(-\mathrm{N}^{+} \mathrm{Me}_{3}\right)$ segments. Oligomers with longer fluoroalkyl segments and greater molar ratios of monomer in monomer/peroxide are likely to have stronger association through aggregation of fluoroalkyl moieties and intermolecular hydrogen bondings between hydroxy or carboxy segments to cause physical gelation. In particular, fluorinated HOPPA oligomers possessing perfluoropropyl and perfluoro-1-methyl-2-oxapentyl segments (No. 10, 11 in Table III) caused gelation in water and DMSO. The corresponding MHPTA oligomers ( No. 2, 3 in Table III) were soluble in these solvents. Hydrogen bonding interactions between hydroxy or carboxy segments in HOPPA oligomers may thus become stronger than those between hydroxy segments in MHPTA oligomers when fluorinated oligomers form gels in these solvents.

Our fluoroalkylated end-capped MHPTA or HOPPA oligomers form gels not only in water but also in organic polar solvents such as $\mathrm{MeOH}$, EtOH, DMF, and DMSO under non-crosslinked conditions, since fluoroalkyl segments are solvophobic in aqueous and organic media, and enhance the aggregation due to the strong interaction between fluoroalkyl end-capped segments in oligomers. Fluoroalkyl segments in the double-chain amphiphiles possessing long perfluoroalkyl chains in the hydrophobic portion such as $\left\{\left[\mathrm{CF}_{3}\left(\mathrm{CF}_{2}\right)_{7}-\mathrm{CH}_{2} \mathrm{CH}_{2} \mathrm{C}(=\mathrm{O}) \mathrm{OCH}_{2}-\right.\right.$ $\left.\left.\mathrm{CH}_{2}\right]_{2} \mathrm{~N}-\mathrm{C}(=\mathrm{O}) \mathrm{CH}_{2} \mathrm{~N}^{+} \mathrm{Me}_{3} \mathrm{Cl}^{-}\right\}$should provide the solvophobic property to form stable bilayer membrances in water and organic solvents. ${ }^{17-21}$ Thus, such unique aggregate of fluoroalkyl segments in these media would be remarkably enhanced due to the stability by the self-organization of oligomers to cause gelation. It was clarified that non-fluorinated MHPTA or HOPPA oligomers do not form gels either in water or polar organic solvents. This suggests that only hydrogen bonding interactions cannot be involved in physical gelation, and the aggregation of fluoroalkylated endcapped segments is essential for gelation.

It is of particular interest to clarify the surface properties of these gelling or gel-like oligomers. Thus, we measured the reduction of surface tension of aqueous solutions of gel-like perfluoro-1-methyl-2-oxapentylated MHPTA oligomers (No. 5 in Table I) with the Wilhelmy plate method at $30^{\circ} \mathrm{C}$. The surface tension of aqueous

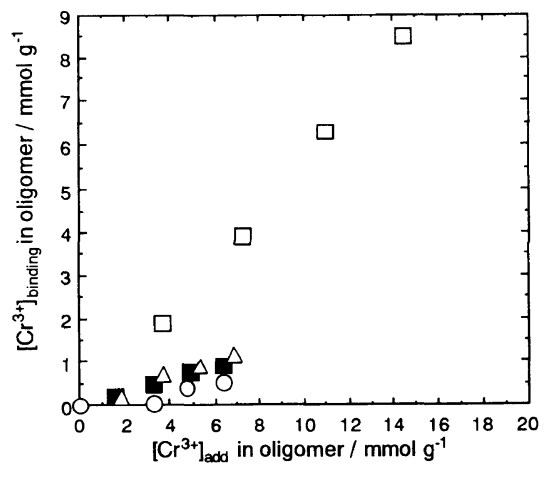

Figure 3. Relationship between relative amounts of $\mathrm{Cr}^{3+}$ binding to fluoroalkylated oligomers and initial $\mathrm{Cr}^{3+}$. $\mathrm{O}, \mathrm{R}_{\mathrm{F}}-(\mathrm{MHPTA})_{n}-\mathrm{R}_{\mathrm{F}}$; $\mathrm{R}_{\mathrm{F}}=\mathrm{CF}\left(\mathrm{CF}_{3}\right) \mathrm{OC}_{3} \mathrm{~F}_{7}$ (No. 6); $\mathbf{\square}, \mathrm{R}_{\mathrm{F}}-(\mathrm{HOPPA})_{n}-\mathrm{R}_{\mathrm{F}} ; \mathrm{R}_{\mathrm{F}}=\mathrm{CF}\left(\mathrm{CF}_{3}\right)-$ $\mathrm{OC}_{3} \mathrm{~F}_{7}$ (No. 13); $\triangle, \mathrm{R}_{\mathrm{F}}-(\mathrm{HOPPA})_{n}-\mathrm{R}_{\mathrm{F}} ; \mathrm{R}_{\mathrm{F}}=\mathrm{CF}\left(\mathrm{CF}_{3}\right) \mathrm{OCF}_{2} \mathrm{CF}\left(\mathrm{CF}_{3}\right)-$ $\mathrm{OC}_{3} \mathrm{~F}_{7}\left(\right.$ No. 16); $\square, \mathrm{R}_{\mathrm{F}}-(\mathrm{MHPTA})_{n}-\mathrm{R}_{\mathrm{F}} ; \mathrm{R}_{\mathrm{F}}=\mathrm{CF}\left(\mathrm{CF}_{3}\right) \mathrm{OC}_{3} \mathrm{~F}_{7}$ (No. 7).

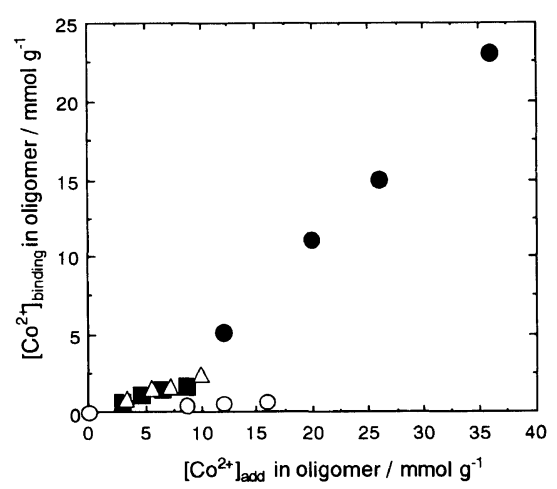

Figure 4. Relationship between relative amounts of $\mathrm{Co}^{2+}$ binding to fluoroalkylated oligomers and initial $\mathrm{Co}^{2+} . \mathrm{O}, \mathrm{R}_{\mathrm{F}}-(\mathrm{MHPTA})_{n}-\mathrm{R}_{\mathrm{F}} ; \mathrm{R}_{\mathrm{F}}=$ $\mathrm{CF}\left(\mathrm{CF}_{3}\right) \mathrm{OC}_{3} \mathrm{~F}_{7}$ (No. 6);, $\mathrm{R}_{\mathrm{F}}-(\mathrm{HOPPA})_{n}-\mathrm{R}_{\mathrm{F}} ; \mathrm{R}_{\mathrm{F}}=\mathrm{CF}\left(\mathrm{CF}_{3}\right) \mathrm{OC}_{3} \mathrm{~F}_{7}$ (No. 13); $\triangle, \mathrm{R}_{\mathrm{F}}$-(HOPPA) ${ }_{n}-\mathrm{R}_{\mathrm{F}} ; \mathrm{R}_{\mathrm{F}}=\mathrm{CF}\left(\mathrm{CF}_{3}\right) \mathrm{OCF}_{2} \mathrm{CF}\left(\mathrm{CF}_{3}\right) \mathrm{OC}_{3} \mathrm{~F}_{7}$ (No. 16); $\mathrm{R}_{\mathrm{F}}-(\mathrm{MHPTA})_{n}-\mathrm{R}_{\mathrm{F}} ; \mathrm{R}_{\mathrm{F}}=\mathrm{CF}\left(\mathrm{CF}_{3}\right) \mathrm{OC}_{3} \mathrm{~F}_{7}$ (No. 7).

solutions of non-fluorinated MHPTA oligomers was measured for comparison. The results are shown in Figure 2.

As shown in Figure 2, significant decrease in the surface tension of water, around $15 \mathrm{~m} \mathrm{~N} \mathrm{~m}^{-1}$, was found for this gel-like fluorinated oligomer in contrast to the corresponding non-fluorinated oligomer. This fluorinated oligomer exhibited a clear break point resembling a CMC (critical micell concentration). This indicates the formation of molecular aggregate, that is, the formation of macromolecular aggregates (gel-like). Thus, these fluoroalkylated end-capped oligomers should interact with aggregations of fluoroalkyl segments as well as have surface active property imparted by fluorine.

Hitherto, much attention has been focused on the uptake or release of metal ions by chemically crosslinked polymer gels. Osada et al. reported the adsorptive properties against metal ions on the swelling equilibrium of polymer gels. ${ }^{22}$ Therefore, it is much interesting to study the adsorptive properties against metal ions on the swelling equilibrium of our new fluoroalkylated endcapped MHPTA or HOPPA oligomer hydrogels. Thus, the uptake and release of $\mathrm{Cr}^{3+}$ and $\mathrm{Co}^{2+}$ by these oligomer hydrogels were studied for a wide range of metal-ion concentrations $\left(15-60 \mathrm{mmol} \mathrm{dm}^{-3}\right)$. The metal-ion concentration of supernatant liquid after the incubation (at $25^{\circ} \mathrm{C}$ for $24 \mathrm{~h}$ ) was spectrophotometrically 
determined from a calibration curve showing the relationship between metal ion concentration and absorbance at $580 \mathrm{~nm}\left(\mathrm{Cr}^{3+}\right)$ or $510 \mathrm{~nm}\left(\mathrm{Co}^{2+}\right)$. The results are shown in Figures 3 and 4, respectively.

As shown in Figures 3 and 4, the uptake of $\mathrm{Cr}^{3+}$ or $\mathrm{Co}^{2+}$ increased linearly with initial concentration of $\mathrm{Cr}^{3+}$ or $\mathrm{Co}^{2+}$. Fluorinated hydrogels possessing lower $C_{\min }$ values (i.e., No. 7: $C_{\min }=7 \mathrm{~g} \mathrm{dm}^{-3}$ ) had stronger binding power with $c a$. $60 \%$ binding ratio [ratio based on relative amount of $\mathrm{Cr}^{3+}$ (or $\mathrm{Co}^{2+}$ ) binding to gel $\left(\left[\mathrm{Cr}^{3+}\right]_{\text {binding }} /\right.$ oligomer $)$ and relative amount of initial $\mathrm{Cr}^{3+}\left(\left[\mathrm{Cr}^{3+}\right]_{\text {add }}\right.$ /oligomer $\left.)\right] . \mathrm{R}_{\mathrm{F}^{-}}(\mathrm{MHPTA})_{n}-\mathrm{R}_{\mathrm{F}}($ No. 6 in Table I) hydrogel possessing higher $C_{\min }$ than No. 7 in Table I had weaker $\mathrm{Cr}^{3+}$ and $\mathrm{Co}^{2+}$ binding power ( $c a$. $10 \%$ and $c a .5 \%$ binding ratios, respectively) under the similar conditions. $\mathrm{R}_{\mathrm{F}}-(\mathrm{HOPPA})_{n}-\mathrm{R}_{\mathrm{F}}($ No. 16 and 13 in Table I) hydrogels had $\mathrm{Cr}^{3+}$ binding power ( $c a .17 \%$ and $c a$. $15 \%$ binding ratios, respectively) as shown in Figure 3, and the hydrogel (No. 16) possessing lower $C_{\min }$ had a slightly higher metal binding power. Similar results for the uptake of $\mathrm{Co}^{2+}$ by $\mathrm{R}_{\mathrm{F}}-(\mathrm{HOPPA})_{n}-\mathrm{R}_{\mathrm{F}}$ were obtained as in Figure 4, and there was no uptake selectivity of the fluorinated hydrogels for these metal ions. From these results, it can be said that the fluoroalkylated end-capped oligomer networks in the hydrogels possessing lower $C_{\min }$ are likely to bind strongly to the metal ion, since the stronger aggregation of fluoroalkyl segments is necessary for establishment of a physical gel network to exhibit lower $C_{\text {min }}$

The release of $\mathrm{Cr}^{3+}$ or $\mathrm{Co}^{2+}$ increases linearly with initial concentration of $\mathrm{Cr}^{3+}$ (or $\mathrm{Co}^{2+}$ ) binding to the hydrogels (data not shown) to afford ca. $60 \%$ releasing ratio [ratio based on the relative amount of $\mathrm{Cr}^{3+}$ (or $\mathrm{Co}^{2+}$ ) released into water and relative amount of $\mathrm{Cr}^{3+}$ (or $\mathrm{Co}^{2+}$ ) binding to gel], respectively, after incubation at $25^{\circ} \mathrm{C}$ for $24 \mathrm{~h}$. On the other hand, $\mathrm{Cr}^{3+}\left(\right.$ or $\mathrm{Co}^{2+}$ ) was not released from $\mathrm{Cr}^{3+}$ (or $\mathrm{Co}^{2+}$ )-bound $\mathrm{R}_{\mathrm{F}^{-}}$ (HOPPA) ${ }_{n}-\mathrm{R}_{\mathrm{F}}$ hydrogels at all under similar releasing conditions. This is because interactions of $\mathrm{Cr}^{3+}\left(\mathrm{or} \mathrm{Co}^{2+}\right)$ and $\mathrm{R}_{\mathrm{F}}-(\mathrm{HOPPA})_{n}-\mathrm{R}_{\mathrm{F}}$ hydrogels are ionic, whereas the main driving force for $\mathrm{Cr}^{3+}$ (or $\mathrm{Co}^{2+}$ ) binding to $\mathrm{R}_{\mathrm{F}}-(\mathrm{MHPTA})_{n}-\mathrm{R}_{\mathrm{F}}$ is coordinate bonds between $\mathrm{Cr}^{3+}$ (or $\mathrm{Co}^{2+}$ ) and $\mathrm{OH}$ segments. The electrostatic repulsion between the cation $\left(\mathrm{N}^{+}\right)$segments in these MHPTA hydrogels and the metal ions may promote the release of metal ions into water.

Very recently, we demonstrated that gelling fluoroalkylated end-capped oligomers containing betaine segments act as potent and selective inhibitors against antihuman immunodeficiency virus (HIV)-1. ${ }^{10}$ Therefore, we would expect our present gelling (or gel-like) fluoroalkylated oligomers to behave as novel polymeric inhibitors of HIV-1 and for this reason such fluoroalkylated MHPTA oligomers have been evaluated for activity against HIV-1 replication in MT-4 cells. However, each oligomer was found inactive against HIV-1 replication, and the oligomers were toxic to the host cells. Previously, we reported that the activity against HIV-1 is sensitive to the oleophilic property of fluoroalkylated end-capped oligomers, and the fluoroalkylated oligomers become more hydrophilic, activity is, in general, not observed. ${ }^{23}$ Hence, that our present oligomers possess no anti-HIV-1 activity would be due to these oligomers having strong hydrophilic trimethylammonium and hydroxy segments.

Our present fluoroalkylated end-capped MHPTA oligomers were toxic to host cells, and thus should show antibacterial activity. They have been evaluated for antibacterial activity against Staphylococcus aures by viable cell counting method as already reported. ${ }^{24}$ $1.5 \times 10^{8}$ cells $\mathrm{ml}^{-1}$ of Staphylococcus aures were exposed to $1 \mathrm{mg} \mathrm{ml}^{-1}$ oligomers in saline, and the colony-forming units (cfu) versus exposure for these oligomers against Staphylococcus aures were as follows:

$$
\begin{aligned}
\mathrm{R}_{\mathrm{F}}-(\mathrm{MHPTA})_{n}-\mathrm{R}_{\mathrm{F}} ; & \\
\mathrm{R}_{\mathrm{F}}=\mathrm{CF}\left(\mathrm{CF}_{3}\right) \mathrm{OCF}_{2} \mathrm{CF}\left(\mathrm{CF}_{3}\right) \mathrm{OC}_{3} \mathrm{~F}_{7}(\mathrm{Run} 8) & 3.3 \times 10^{5} \mathrm{cfu} \\
\mathrm{R}_{\mathrm{F}}=\mathrm{CF}\left(\mathrm{CF}_{3}\right) \mathrm{OCF}_{2} \mathrm{CF}\left(\mathrm{CF}_{3}\right) \mathrm{OC}_{3} \mathrm{~F}_{7} \text { (Run 9) } & 2.1 \times 10^{7} \mathrm{cfu} \\
\mathrm{R}_{\mathrm{F}}-(\mathrm{MHPTA})_{x}-\left(\mathrm{CH}_{2}-\mathrm{CHSiMe}\right)_{y}-\mathrm{R}_{\mathrm{F}} ; & \\
\mathrm{R}_{\mathrm{F}}=\mathrm{CF}\left(\mathrm{CF}_{3}\right) \mathrm{OCF}_{2} \mathrm{CF}\left(\mathrm{CF}_{3}\right) \mathrm{OC}_{3} \mathrm{~F}_{7} \text { (Run 18) } & 1.1 \times 10^{5} \mathrm{cfu}
\end{aligned}
$$

Fluoroalkylated end-capped MHPTA homo- and co-oligomers showed bacterial activity to some extent ( $-10^{5}$ colony forming units levels). In these oligomers, fluoroalkylated MHPTA-trimethylvinylsilane co-oligomer was the most active, with $1.1 \times 10^{5} \mathrm{cfu}$.

Fluoroalkylated end-capped oligomers containing hydroxy segments thus cause gelation under the noncrosslinked conditions, especially the aggregation of fluoroalkyl units in water and/or in organic media becomes a new driving factor for gelation as well as interactions such as hydrogen bonding and ionic interaction. Fluoroalkylated MHPTA oligomers had high metal-binding or releasing power. Our gelling or gel-like fluoroalkylated MHPTA oligomers exhibited not only the properties imparted by fluorine such as surface activity, but also some antibacterial activity. Therefore, our present fluorintaed oligomer gels should be used in various fields as novel fluorinated functional materials.

Acknowledgment. This work was partially supported by a Grant-in-Aid for Scientific Research No. 09650945 from the Ministry of Education, Science, Sports and Culture of Japan, for which the authors are grateful.

\section{REFERENCES}

1. (a) R. E. Banks, B. E. Smart, and J. C. Tatlow, Ed., "Organofluorine Chem.," Plenum, New York, N.Y., 1994; (b) Z.-Y. Yang, A. E. Feiring, and B. E. Smart, J. Am. Chem. Soc., 116, 4135 (1994).

2. H. Sawada, Chem. Rev., 96, 1779 (1996)

3. J. F. Elman, B. D. Johs, T. E. Long, and J. T. Koberstein, Macromolecules, 27, 5341 (1994).

4. S. Affrossman, M. Hartshorne, T. Kiff, R. A. Pethrick, and R. W. Richards, Macromolecules, 27, 1588 (1994).

5. M. O. Hunt, Jr., A. M. Belu, R. W. Linton, and J. M. DeSimone, Macromolecules, 26, 4854 (1993).

6. S. Affrossman, P. Bertrand, M. Hartshorne, T. Kiff, D. Leonard, R. A. Pethrick, and R. W. Richards, Macromolecules, 29, 5432 (1996).

7. B. Xu, L. Li, A. Yekta, Z. Masoumi, S. Kanagalingam, M. A Winnik, K. Zhang, and P. M. Macdonald, and S. Menchen, Langmuir, 13, 2447 (1997).

8. J. Wang, G. Mao, C. K. Ober, and E. J. Kramer, Polym. Prepr., Am. Chem. Soc., Div. Polym. Chem., 38, 953 (1997).

9. Z. Su, D. Wu, S. L. Hsu, and T. J. McCarthy, Polym. Prepr., Am. Chem. Soc., Div. Polym. Chem., 38, 951 (1997).

10. H. Sawada, S. Katayama, Y. Nakamura, T. Kawase, Y Hayakawa, and M. Baba, Polymer, 39, 743 (1998).

11. H. Sawada, Y. Nakamura, S. Katayama, and T. Kawase, Bull. Chem. Soc. Jpn., 70, 2839 (1997). 
12. R. J. Twieg, T. P. Russell, R. Siemens, and J. F. Rabolt, Macromolecules, 18, 1361 (1985).

13. H. Sawada, T. Tanimura, S. Katayama, and T. Kawase, J. Chem. Soc., Chem. Commun., 1391 (1997).

14. (a) H. Sawada and M. Nakayama, J. Fluorine Chem., 51, 117 (1990); (b) H. Sawada, M. Yoshida, H. Hagii, K. Aoshima, and M. Kobayashi, Bull. Chem. Soc. Jpn., 59, 215 (1986).

15. (a) K. Hanabusa, R. Tanaka, M. Suzuki, M. Kimura, and H. Shirai, Adv. Mater., 9, 1095 (1997); (b) K. Hanabusa, K. Okui, K. Karaki, M. Kimura, and H. Shirai, J. Colloid Interface Sci., 195, 86 (1997).

16. K. Hanabusa, C. Koto, M. Kimura, H. Shirai, and A. Kakehi, Chem. Lett., 429 (1997).

17. T. Kunitake, Y. Okahata, and S. Yasunami, J. Am. Chem. Soc., 104, 5547 (1987).
18. T. Kunitake and N. Higashi, J. Am. Chem. Soc., 107, 692 (1985)

19. T. Kunitake and N. Higashi, Macromol. Chem. Phys. Suppl., 81 (1985).

20. Y. Ishikawa, H. Kuwahara, and T. Kunitake, Chem. Lett., 1737 (1989).

21. Y. Ishikawa, H. Kuwahara, and T. Kunitake, J. Am. Chem. Soc., 111, 8530 (1989).

22. Y. Osada and M. Takase, Nippon Kagaku Kaishi, 439 (1983).

23. H. Sawada, K. Tanba, N. Itoh, C. Hosoi, M. Oue, M. Baba, T. Kawase, M. Mitani, and H. Nakajima, J. Fluorine Chem., 77, 51 (1996).

24. (a) H. Sawada, S. Katayama, M. Oue, T. Kawase, Y. Hayakawa, M. Baba, T. Tomita, and M. Mitani, J. Jpn. Oil Chem. Soc., 45 161 (1996). (b) H. Sawada, K. Tanba, T. Tomita, T. Kawase, M. Baba, and T. Ide, J. Fluorine Chem., 84, 141 (1997). 